astrocyte-

conditioned

medium

induced

downregulation

of $\mathrm{MHCl}$ in

motor neurons

MOTOR NEURON DISEASE

\title{
Loss of $\mathrm{MHCl}$ exposes motor neurons to astrocyte-mediated toxicity
}

A report in Nature Medicine sheds new light on astrocyte-mediated toxicity, which is thought to be an important contributor to motor neuron death in amyotrophic lateral sclerosis (ALS). The study demonstrates that astrocytes carrying ALS-related mutations cause downregulation of MHC class I (MHCI) expression on motor neurons, which in turn makes the neurons more susceptible to the toxic effects of the astrocytes.

These findings reconcile two pre-existing lines of evidence. First, astrocytes from individuals with ALS have been shown to be toxic to motor neurons in culture. Second, in the $S O D 1^{\mathrm{G} 93 \mathrm{~A}}$ mouse model of ALS, elevated MHCI expression on motor neurons is associated with reduced rates of disease progression.

"We have always been interested in understanding the mechanisms underlying the astrocyte-motor neuron interaction," says Brian Kaspar, who led the new study. "Furthermore, we have been studying immune molecules in the context of glial cell biology in ALS."
Kaspar's team observed loss of MHCI expression in spinal motor neurons from SOD $1^{\mathrm{G} 93 \mathrm{~A}}$ mice and patients with ALS. In addition, wild-type motor neurons that were co-cultured with astrocytes from $S O D 1^{\mathrm{G} 93 \mathrm{~A}}$ mice exhibited downregulation of MHCI.

Interestingly, SOD $1^{\mathrm{G} 93 \mathrm{~A}}$ motor neurons that were cultured in the absence of $S O D 1^{\mathrm{G} 93 \mathrm{~A}}$ astrocytes expressed near-normal levels of $\mathrm{MHCI}$, and SOD $1^{\mathrm{G} 93 \mathrm{~A}}$ astrocyteconditioned medium induced downregulation of MHCI in motor neurons. Taken together, these findings imply that factors secreted by $S O D 1^{\mathrm{G} 93 \mathrm{~A}}$ astrocytes were responsible for the loss of MHCI expression.

To explore the relationship between MHCI expression and vulnerability to astrocyte-mediated toxicity, the researchers generated motor neuron lines that overexpressed various MHCI molecules, and they co-cultured these cells with $S O D 1^{\mathrm{G} 93 \mathrm{~A}}$ astrocytes. $\mathrm{H} 2-\mathrm{K}^{\mathrm{b}}$ overexpression was found to provide complete protection against
$S O D 1^{\mathrm{G} 93 \mathrm{~A}}$ astrocyte-induced toxicity, whereas two other MHCI molecules, $\mathrm{H} 2-\mathrm{D}^{\mathrm{b}}$ and $\mathrm{H} 2-\mathrm{L}^{\mathrm{d}}$, only provided partial protection.

The team also generated $S O D 1^{\mathrm{G} 93 \mathrm{~A}}$ mice that overexpressed $H 2^{k}$ or $\mathrm{H}_{2}{ }^{d}$ - the genes that encode $\mathrm{H} 2-\mathrm{K}^{\mathrm{b}}$ and $\mathrm{H} 2-\mathrm{D}^{\mathrm{b}}$, respectively. Consistent with the in vitro data, overexpression of $H 2^{k}$, but not $H 2^{d}$, delayed disease progression and improved motor performance.

"Further work is warranted; for example, what are astrocytes secreting - or not secreting - that has the effect of reducing MHCI presentation on motor neurons?" says Kaspar. Therapeutic strategies for future investigation could include maintenance of MHCI expression on motor neurons, as well as inhibition of astrocyte-induced toxicity.

Heather Wood

ORIGINAL ARTICLE Song, S. et al. Major histocompatibility complex class I molecules protect motor neurons from astrocyte-induced toxicity in amyotrophic lateral sclerosis. Nat. Med. http://dx.doi.org/10.1038/nm.4052 